\title{
El paradigma de la sostenibilidad: Gobernanza global y el modelo europeo de "desarrollo sostenible"
}

\author{
María Dolores Sánchez Galera*
}

\section{Resumen}

Nuestra discusión pretende analizar los retos conceptuales que se esconden detrás del paradigma de la sostenibilidad, para entender más adelante en nuestro análisis el compromiso jurídico efectivo de la UE a la hora de implementar las políticas de desarrollo sostenible y las medidas legales que le siguen, que serían difíciles de entender sino se acude a las pautas de desarrollo sostenible que ha lanzado la gobernanza global. Para ello, nuestro prisma analítico tocará los límites del tan mencionado -en los últimos añosderecho global. Las medidas legislativas de la UE en el campo de la "sostenibilidad" sólo pueden entenderse a la luz del paralelo esfuerzo de determinación de conceptos, categorías y principios básicos que los acuerdos internacionales, en aras del desarrollo de un principio de justicia intergeneracional y desde la óptica del cambio climático, han impulsado. Con independencia de la dificultad que comporta la inexistencia de consenso sobre el significado de la expresión "desarrollo sostenible", partimos de la base que el TFUE y el TUE nos dan las pautas para una interpretación de un modelo conceptual de desarrollo sostenible, como nos lo dio en su día el Informe Brundtland. Podemos afirmar que Europa cuenta hoy con un acquis communautaire de sostenibilidad que ya es innegable.

\section{Palabras clave}

Sostenibilidad; Derecho Global; Cambio Climático; protección ambiental; gobernanza global.

\section{TITLE}

The paradigm of "sustainability": Global governance and the European model of "sustainable development"

\section{Abstract}

This article seeks to analyze the conceptual challenges embedded in the global legal system's sustainability paradigm. Our discussion focuses on the European Union's legal commitment for the implementation of sustainable development policies. It would be difficult to understand it without paying attention to the global context and the sustainable development guidelines under the so called "global governance". To do this, we have set up an analytical framework that assesses the parameters of global law - a pivotal reference in legal discourse in recent years. EU legislation within the field of "sustainability" can only be understood in light of the parallel effort, via international agreements, for the definition of concepts, categories and basic principles upon which we can ultimately formulate an intergenerational justice principle. Furthermore, as proposed by the Brundtland Report, despite the difficulties arising from the lack of consensus on the meaning of "sustainable development", the TFEU and the TEU provide guidelines for a stronger interpretation of a conceptual model of sustainable development. In this way, it is possible to argue that Europe has now an undeniable sustainability acquis communautaire.

\section{KEYWORDS}

Sustainability; Global law; climate change; environmental protection: global governance.
* María Dolores SÁNCHEZ

GALERA, Marie Curie-Conex Fellow-profesora asistente a la Universidad Carlos III de Madrid. Ha sido investigadora del Instituto de Derecho Internacional de La Haya y sigue colaborando con la red "Rule of Law" dirigida por el Instituto. Profesora de Derecho Europeo y Derecho Ambiental en programas de pregrado y postgrado desde 2007. Colabora como investigadora independiente para diferentes fundaciones y organizaciones benéficas y fue funcionaria de cooperación internacional para Caritas Italiana por un par de años. Antes de incorporarse a UC3M, fue investigadora del Instituto DIRPOLIS en la Scuola Superiore Sant'Anna, Pisa, Italia.

Recibido:

2016-09-06

Aceptado:

2017-02-13

DOI: $10.15366 /$

relacionesinternaci onales2017.34.001 


\section{ntroducción}

Los rápidos cambios sufridos por el panorama global desde el punto de vista del "uso" y "abuso" de los recursos naturales y los conflictos de naturaleza ambiental de escala mundial, que condicionan la evolución del planeta en general, han contribuido a la difusión multidimensional de la noción de "sostenibilidad" y al de la gobernanza global. Fruto de este enfoque integral e integrado del desarrollo y de los problemas ambientales y climáticos que ha generado y genera la acción del hombre, así como del abuso de los recursos naturales, surge el "derecho medioambiental global". Éste se utiliza cada vez más para cuestionar el paradigma interestatal de la protección ambiental internacional y determina el auge de un proceso de globalización en el modo de entender y querer afrontar las dificultades no sólo ambientales, sino sociales, constitucionales, económicas y políticas que son ya parte de un mismo problema ${ }^{1}$.

La sostenibilidad nace como un concepto inclusivo y flexible, así como evasivo, aún no claramente clasificado en el marco de la gobernanza ambiental. No obstante es lo suficientemente importante como para guiar la creación de leyes internacionales a todos los niveles y para guiar el creciente papel de las instituciones internacionales. Sostenibilidad es sin duda un término, hoy en día, objeto de amplias especulaciones; no obstante necesita todavía ser constantemente analizado dentro de parámetros que valorizan la naturaleza evolutiva y abstracta de dicha noción, y la contextualizan para dar la fuerza legal que le dote de eficiencia y aplicabilidad. La gobernanza europea despliega su propio poder de acción siguiendo las líneas globales de acción. Europa es un fantástico laboratorio de gobernanza sostenible a través de un complejo paquete normativo y político que aborda la energía, la protección del clima y la gobernanza sostenible en su conjunto protegiendo el interés común de la humanidad. Por lo tanto, la UE va más allá de los logros del principio de integración, que desempeñó el papel más importante en el pasado a la hora integrar la política ambiental en otras políticas sectoriales, para abordar la prevaricación intergeneracional de nuestra era². Ocuparse de sostenibilidad es relevante porque es, y seguirá siendo, un eje central de la evolución humana en su conjunto.

Hoy, la necesidad de una transición energética para un uso eficiente de nuestros recursos naturales, y la lucha contra el cambio climático lideran la esfera de la protección ambiental que deriva de la hegemonía de la noción de sostenibilidad. El concepto ha imbuido la política en todas sus dimensiones y el "desarrollo sostenible" se ha convertido hoy en día en un objetivo político de primera magnitud tanto en el plano internacional, como en el estatal y el local, implicando un enfoque integral de los procesos económicos, políticos y sociales.

El presente trabajo abordará algunos aspectos conceptuales relacionados con el paradigma de la sostenibilidad y los desafíos que plantea en el contexto internacional. Es

1 Veáse, MORGERA, Elisa, "Bilateralism at the Service of Community Interests? Non-judicial Enforcement of Global Public Goods in the Context of Global Environmental Law" en The European Journal of International Law, vol. 23, n० 3, 2012, p. 745.

2 El debate contemporáneo sobre la naturaleza y la influencia humana en relación con la naturaleza ha sido marcado por la emergencia de una hipótesis geológica que ha ganado terreno rápidamente fuera de la esfera de las ciencias naturales, se trata de la llamada era del Antropoceno. De acuerdo con el "Antropoceno" el cambio físico de la tierra - esto es, biodiversidad, clima, etc. - está causado fundamentalmente por la actividad del hombre. Somos los causantes de nuestras catástrofes globales. Veáse, ARIAS MALDONADO, Manuel, Environment and Society: Socionatural relations in the Anthropocene, Springer, 2015. 
importante asumir que el contexto global, específicamente el derecho ambiental global situado dentro del contexto de las interacciones entre una pluralidad de órdenes legales determina y determinará en manera siempre creciente la existencia constitucional de la sostenibilidad, no sólo en nuestro ordenamiento jurídico de análisis, la UE, sino en general en el entramado jurídico internacional.

El cambio climático, los derechos procesales medioambientales y el crecimiento económico en aras de la sostenibilidad se colocan a la cabeza de los discursos de las ciencias sociales creando sinergias entre sus diferentes pero ya interconectados discursos científicos para implementar leyes y políticas públicas capaces de proporcionar mecanismos de protección y aplicación de un desarrollo sostenible a nivel global. Como señala, Rodrigo Hernández

"El Derecho Internacional puede desempeñar un papel muy importante para lograr el objetivo del desarrollo sostenible porque, por un lado, proporciona un sistema válido para consolidar un enfoque integrado del medio ambiente y del desarrollo por medio de principios y de normas generales y, por otro lado, puede ofrecer el marco regulador concreto para la cooperación entre todos los actores relevantes" ${ }^{\prime \prime}$.

Así, el presente trabajo se estructurará a lo largo de tres partes. Las primeras dos partes tratarán las cuestiones más conceptuales y doctrinales de este trabajo. Se centrarán en el contenido de dos nuevos paradigmas importantes de las ciencias jurídicas, la sostenibilidad y la gobernanza global. Se introduce un análisis de los conceptos de "sostenibilidad" y "desarrollo sostenible", así como de su marco normativo y las consecuencias de la gobernanza multinivel a nivel internacional. Se hará hincapié en el derecho ambiental global y su influencia creciente en los ordenamientos nacionales con la "emergencia de un derecho administrativo mundial" que acuña la nueva noción de sostenibilidad de naturaleza hegemónica en las políticas internacionales, nacionales, regionales y locales. La opinión del autor es, que los diferentes niveles de gobernanza global hoy, determinan los fundamentos para la comprensión de esta noción evolutiva de sostenibilidad y de la protección ambiental más allá de las fronteras estatales. La última parte del documento establecerá las bases constitucionales y los fundamentos políticos para la interpretación del marco jurídico de la "sostenibilidad" y las políticas de "desarrollo sostenible" en el contexto europeo que vienen obviamente determinadas por el armazón institucional y político global.

\section{Una breve historia de sostenibilidad: La relación entre "Sostenibilidad" y "Desarrollo sostenible"}

El "desarrollo sostenible", del cual podríamos decir que es el nuevo paradigma de nuestra era, potencialmente podría abrazar indistintamente cada campo de acción y, como Jeffrey Sachs expresa, es una "forma de entender el mundo y un método para resolver problemas

3 RODRIGO, Ángel J., "El principio de integración de los aspectos económicos, sociales y medioambientales del desarrollo sostenible" en Revista Española de Derecho Internacional, vol. LXIV, no 2, 2012, p. 134. Véase también, SANDS, Peel, "Environmental Protection in the Twentieth Century: Sustainable Development and International Law" en VIG, Norman J. y AXELROLD, Regina S. (eds.), The Global Environment. Institutions, Law and Policy, Earthscan, Washington D.C., 1999, ps. 116-137. 
globales"4. Sin embargo, la idea de sostenibilidad es tan antigua como la existencia humana ${ }^{5}$ De hecho, antes de comprometerse más con los orígenes del concepto de sostenibilidad, es importante dedicar nuestra atención a la diferenciación entre sostenibilidad y desarrollo sostenible. Ambos términos son hoy en día utilizados indistintamente por las ciencias sociales aunque tengan significados distintos.

La noción de sostenibilidad precede a la de desarrollo sostenible. La noción de desarrollo sostenible nace sólo a finales del siglo XX6 . La sostenibilidad se centra en la "capacidad de los seres humanos para vivir dentro de las limitaciones ambientales"7 y el corazón del concepto es el respeto de los límites ecológicos y la integridad ecológica. Es importante señalar que la sostenibilidad precede también al carácter normativo del desarrollo sostenible, su existencia pertenece a nuestra era. En cuanto a la evolución histórica del término sostenibilidad, debemos decir que el término surgió por primera vez durante la época de la Ilustración. El telón de fondo con el que se acuñó el término sostenibilidad fue la experiencia de una crisis ecológica que consistió en la deforestación causada por el rápido aumento de la demanda económica.

No por casualidad, el concepto adopta su nombre a principios del siglo XVIII de la mano de un burócrata sajón Hans Carl von Carlowitz que trabajaba en el sector minero que acuñó el término "Nachhalligkeit" para describir la práctica de cosechar madera continuamente en el mismo bosque 9 . Pero, de hecho, la silvicultura de rendimiento sostenido en el tiempo tomó forma en este momento, no sólo en Europa Occidental, sino también en Japón, alrededor de las otras partes de Asia y en las islas Coloniales, así como en las Indias Occidentales y Orientales ${ }^{10}$. Así es como entre los teóricos de gestión forestal del siglo XIX emerge la visión compartida de "mantener" la vida en su conjunto para facilitar la evolución humana. Esta visión se limitaba a académicos y academias forestales en Alemania, pero las academias forestales de otros lugares siguieron el mismo contexto ecológico. Es así que, durante el siglo XIX el concepto surgió como término central dentro de la ciencia forestal, creciendo ampliamente para incluir todo el espectro del ecosistema "bosque", que comprende ubicaciones, fertilidad de los suelos, diversidad de organismos, hábitat para la vida silvestre, reservorio de agua, protección contra la erosión y el espacio recreativo. Esas fueron las primeras huellas de un

4 SACHS, Jeffrey D., The Age of Sustainable Development, Columbia University Press, Nueva York, 2015, p. 9.

5 Véase en este sentido, CARADONNA, Jeremy L., Sustainability. A History, Oxford University Press, Oxford, 2014.

6 BOSSELMANN, Klaus, "Sustainability and the Courts: A Journey Yet to Begin?" en Journal of Court Innovation, vol. 3, no 1,2010 , p. 338.

7 ROBINSON, John, "Squaring the Circle? Some thoughts on the Idea of Sustainable Development" en Ecological Economics, vol. 48, no 4, 2004, p. 370.

8 Como Bosselmann explica citando los trabajos de los naturalistas Alemanes y Británicos, "[b]y 1650 widespread shortages of wood began to cripple the economies in European countries. At the same time, the new discipline of forest science and management emerged. Its focus was on studying the conditions for sustained forestry and sustainable yield", BOSSELMAN, Klaus, The Principle of Sustainabaility. Transforming Law and Governance, Ashgate, Farnham, 2008, ps. 22-23; con referencia a los trabajos de RADKAU, Joachim, Natur und Macht. Eine Weltgeschichte der Umwelt, Beck, Munich, 2000, p. 245; EVELYN, J, Sylva, or a Discourse of Forest-Trees and the Propagation of Timber in His Majesties Dominions, London, 1664, p. 279.

9 Carlowitz se dio cuenta de la dependencia de la actividad minera de sus recursos naturales básicos y un año antes de su muerte publicó su trabajo recogiendo sus experiencias profesionales y vitales. CARLOWITZ, Hans C. von, Sylvicultura oeconomica, oder haußwirthliche Nachricht und Naturmäßige Anweisung zur wilden BaumZucht, 1713 [edición de Leipzig, repr. Freiberg, TU Bergakademie Freiberg und Akademische Buchhandlung, 2000].

${ }^{10}$ CARADONNA, Jeremy L., Sustainability...op.cit., p. 21. 
enfoque holístico de la sostenibilidad que tenía en su núcleo central un fuerte "contenido ecológico" y que daba las herramientas de guía adecuadas a los gerentes forestales de ese tiempo. Hoy la situación es bastante diferente pero se nutre de ese mismo contenido holístico. Nuestro mundo industrializado y globalizado ofrece un escenario bastante complejo en el que, a pesar de las diferentes estrategias existentes para implementar políticas bajo el paradigma de la sostenibilidad, existe consenso sobre el poder "redentor" de la actuación en aras de la misma, independientemente de la falta de acuerdo sobre su significado.

Lo que hace que la sostenibilidad haya superado al concepto de protección ambiental es el enfoque proactivo de su existencia -independientemente de sus complejidades-, enfocado en la acción en contraposición al carácter defensivo que el derecho ambiental y las medidas ambientales comportan. Los parámetros del desarrollo sostenible, que surgen como la principal expresión normativa y política de la sostenibilidad lanzados por la comunidad internacional en su conjunto, incluyen dentro de un corazón normativo ecológico la justicia social, la equidad, la erradicación de la pobreza, la cultura y la seguridad -entre otros valores globales-. Tal contenido ecológico abarca el derecho de las generaciones futuras y la justicia social con un papel específico y prominente para la orientación de las políticas sectoriales.

\section{Desarrollo sostenible: sus complejidades normativas como concepto dinámico y multidimensional}

Hoy día, las amenazas ambientales están surgiendo desde varios frentes, el paradigma del desarrollo sostenible está consagrado por un número creciente de tratados, constituciones y acuerdos internacionales. Ha alcanzado un valor normativo en el orden global, proponiendo un concepto intergeneracional de desarrollo ${ }^{11}$. El Informe Brundtland de 1987 es de crucial importancia, ofreciendo las bases de la concepción normativa actual del desarrollo sostenible ${ }^{12}$. Es importante hacer hincapié en él porque se consideró vago en los detalles y sin propuestas políticas significativas, pero esta vaguedad ha demostrado ser una fuerza, ya que ha permitido un debate político crucial ${ }^{13}$. El Informe Brundtland proponía el siguiente punto de vista:

"La ecología y la economía están cada vez más entrelazadas a nivel local, regional, nacional y mundial en una red de causas y efectos... [y] ...la pobreza es una de las principales causas de la contaminación ambiental mundial. Es inútil tratar de abordar los problemas ambientales sin una perspectiva más amplia que abarque los factores subyacentes a la pobreza mundial y la desigualdad internacional".

\footnotetext{
${ }^{11}$ La Comisión Brundtland introdujo el componente "intergeneracional" en la clásica definición del concepto de desarrollo sostenible: "Sustainable development is development that meets the needs of the present without compromising the ability of future generations to meet their own needs", en COMISIÓN MUNDIAL SOBRE MEDIO AMBIENTE Y DESARROLLO (UNWCED), Nuestro Futuro Común. Brundtland Report, Oxford University Press, Oxford y Nueva York, 1987, p. 43. Esencialmente el informe Burndtland es un apelo por una justicia distributiva entre ricos y pobres, la población actual y la futura, la humanidad y la naturaleza.

12 Veáse, RODRIGO, Ángel J., El desafío del desarrollo sostenible. Los principios de Derecho internacional relativos al desarrollo sostenible, Centro de Estudios Internacionales, Marcial Pons, Madrid, 2015, p. 9. El autor lleva a cabo una excelente génesis del origen de la formulación de desarrollo sostenible, afirmando que dadas las limitaciones del concepto tradicional del desarrollo económico y la protección ambiental, "el desarrollo sostenible era un oportuno hallazgo diplomático para intentar armonizar las aspiraciones de los Estados en vías de desarrollo en materia de desarrollo económico con las preocupaciones de los estados desarrollados para la protección del medio ambiente".

13 BARNES, Pamela M. y HOERBER, Thomas, C., "Linking the discourse on Sustainability and Governance" en BARNES, Pamela, M. y HOERBER, Thomas, C. (eds) Sustainable development and Governance in Europe, Routledge, Londres y Nueva York, 2013.
} 
Este punto de vista ofrece la posibilidad de una nueva era de crecimiento económico, en contraste con la opinión presentada por aquellos que apoyaron la las declaraciones expuestas en el informe del Club de Roma de 1972 y otros informes y análisis similares. La famosa Declaración de Principios de la Conferencia de Río de 1992 lanzó la noción de desarrollo sostenible en el ámbito jurídico ${ }^{14}$, y aunque los principios de la Declaración de Río no son vinculantes, fueron proclamados en fuertes términos jurídicos.

No fue hasta 1997 que la Asamblea General de la ONU afirmó que la protección ambiental, el desarrollo económico y el desarrollo social eran tres dimensiones interdependientes del desarrollo sostenible ${ }^{15}$, también nominadas: "ecología, economía y equidad". La concepción de los tres pilares del desarrollo sostenible fue confirmada en 2002 en la Cumbre Mundial sobre el Desarrollo Sostenible (CMDS) de Johannesburgo ${ }^{16}$, pero impulsada con éxito por la Declaración de Río de 2012. El instrumento más relevante para su existencia normativa originaria es la 70a Conferencia de la Asociación de Derecho Internacional (ILA) celebrada en Nueva Delhi en 2002. Nos dio la base de una fuerte aceptación doctrinal del desarrollo sostenible como un objetivo global, señalando que el concepto ha sido ampliamente reconocido en varios instrumentos jurídicos nacionales, incluido el derecho de los tratados y la jurisprudencia a nivel internacional y nacional ${ }^{17}$. Con esta declaración, la ILA esperaba contribuir al desarrollo de un "derecho internacional equilibrado y amplio sobre el desarrollo sostenible", como se pide en el Principio 27 de la Declaración de Río sobre el Medio Ambiente y el Desarrollo y el Programa 21 - Conferencia de las Naciones Unidas sobre el Medio Ambiente y el Desarrollo, 1992-.

Hoy, el desarrollo sostenible es uno de los objetivos políticos básicos de la ONU, junto con la paz, la seguridad internacional y la protección de los Derechos Humanos ${ }^{18}$. Esta política global alcanza su momento de gloria el 25 de Septiembre de 2015, con el documento Transforming our World: Agenda 2030 for Sustainable Development. Todos los países han adoptado un conjunto de objetivos -17 objetivos del desarrollo- para erradicar la pobreza, proteger el planeta, y asegurar prosperidad a toda la humanidad como parte de una nueva agenda de desarrollo sostenible. Un elemento clave de estos 17 objetivos del desarrollo (ODS), que deben ser alcanzadas en los próximos 15 años, es que cada uno debe cumplir con su parte: los gobiernos, el sector privado, la sociedad civil y los individuos ${ }^{19}$. Dicho marco de actuación crea las bases para una efectiva gobernanza multinivel cuyo objetivo es la resolución eficaz a nivel local de problemas que son crecientemente de naturaleza global.

14 COMISIÓN MUNDIAL SOBRE MEDIO AMBIENTE Y DESARROLLO (UNWCED), Nuestro Futuro Común...op.cit., ps. 20-21.

${ }^{15}$ Resolución S-19/2 de la Asamblea General de la ONU, 28 de junio de 1997.

${ }^{16}$ La cumbre mundial habló de, "integración de los tres components del desarrollo sostenible-desarrollo económico, desarrollo social y protección ambiental-como pilares de protección interdependientes y de refuerzo mutuo", Informe de la Cumbre Mundial Sobre Desarrollo Sostenible, Johannesburgo, 26 de Agosto al 4 de Septiembre de 2002. Disponible en http://www.cepal.org/rio20/noticias/paginas/6/43766/WSSD_Informe.ESP.pdf [consultado el 27 de Enero de 2017]. Para una lectura detallada véase, LOPERANA, Demetrio, Desarrollo Sostenible y Globalización, Aranzadi, 2003, p. 70.

${ }^{17}$ El texto de esta Declaración, apareció en inglés y en francés, como UN Doc. A/CONF.199/8, 9 Agosto 2002. Véase también la página web ILA: www.ila-hg.org.

18 RODRIGO, Ángel J., El desafío del desarrollo sostenible....op.cit., p.34.

${ }^{19}$ Disponible en http://www.un.org/sustainabledevelopment/ [consultado el 19 de diciembre de 2016]. 
Esto nos lleva a la cuestión - todavía - no resuelta - pero también sin importancia en lo que se refiere a su dimensión práctica y normativa actual- de la naturaleza "multidimensional" de la noción de desarrollo sostenible. De hecho, durante su evolución reciente entendemos que puede ser un objetivo político, un concepto jurídico, un derecho humano, un marco metodológico para la creación y aplicación de políticas públicas y normas internacionales o incluso un instrumento hermenéutico para la interpretación jurídica. Según Ángel J. Rodrigo, como principio jurídico de derecho internacional de naturaleza sustancial, procesal o intersticial, el desarrollo sostenible podría cumplir con una miríada de funciones diferentes ${ }^{20}$. De hecho, su dimensión social que es un requisito abierto de desarrollo, puede estar acompañado de dimensiones de paz, seguridad e incluso cultura. La mayoría de la doctrina internacional considera que los tratados internacionales deben ser interpretados a la luz de estos principios, sin tener en cuenta la línea doctrinal que lo considera una norma consuetudinaria del derecho internacional ${ }^{21}$. Por lo tanto, los componentes esta noción multidimensional, no son tres, y una gobernanza eficaz se está consagrando como elemento clave que transforma el desarrollo sostenible en una guía para la futura gobernanza global. La naturaleza multidimensional del concepto ha ayudado a los gobiernos nacionales y regionales a adecuar sus diferentes políticas a una gobernanza fuerte y multifacética.

De hecho, la buena gobernanza es evidentemente el cuarto componente de la sostenibilidad, e incluye todos los niveles de gobierno. Fue proclamada con fuerza por la ya mencionada Declaración de Delhi (2002) por la ILA, y posteriormente ha sido reafirmada por las Naciones Unidas (2014). La "buena gobernanza" como objetivo político se ha consagrado más recientemente en la Agenda 2030 para el desarrollo sostenible de las Naciones Unidas, así como a nivel regional en la Unión Europea. No se refiere sólo a los gobiernos, y se relaciona con actores privados globales, partes interesadas, multinacionales que actúan en el escenario global y afectan a nuestro mundo viviente. El desafío en juego ahora es la existencia fragmentada de una buena gobernanza en un mundo que no pretende abandonar el control soberano de sus recursos naturales, en riesgo debido a la cantidad de multinacionales y actores sociales del mundo de los negocios que persiguen sus intereses. Corremos el riesgo de abrazar simplemente un "Green developmentalism" que se esfuerza por dar soluciones orientadas al mercado y desarrollo tecnológico que deja intactos los actuales modelos de producción y gobernanza, incluso en el llamado orden jurídico global22. En este caso, es importante determinar la dimensión jurídica del concepto para identificar las funciones interpretativas del desarrollo sostenible que parecen encajar dentro de las categorías tradicionales de normatividad en el ámbito internacional ${ }^{23}$. De hecho, según Klaus Bosselmann, la sostenibilidad como norma "puede ser formulada como la obligación de promover la prosperidad económica a largo plazo y la justicia social dentro de los límites de

20 Véase RODRIGO, Ángel J., El desafío del desarrollo sostenible.... op.cit., p. 34.

${ }^{21}$ Véase, BOYLE, Alan y FREESTONE, David (eds) International Law and Sustainable Development. Past Achievements and Future Challenges, Oxford University Press, Oxford, 1999; SANDS, Peel, "Environmental Protection in the Twentieth Century... op.cit.; FRENCH, Duncan, International Law and Policy of Sustainable Development, Manchester University Press, Manchester, 2005; RODRIGO Ángel J., "El principio de integración de los aspectos económicos, sociales... op.cit., p. 2.

22 Véase, McAFFE, Kathleen, "Selling nature to save it? Biodiversity and the rise of green developmentalism" en Environment and Planning, vol. 17, n० 2, 1999, ps. 133-154.

${ }^{23}$ En este punto veáse, BARRAL, Virginie, "Sustainable Development in International Law: Nature and Operation of an Evolutive Legal Norm" en European Journal of International Law, vol. 23, n 2, ps. 377-400. 
la sostenibilidad ecológica"24. Por lo tanto, de acuerdo con esto, la sostenibilidad entraría en la categoría normativa de los principios legales aunque no haya sido reconocida como tal por el derecho internacional25.

Así pues, podríamos afirmar compartiendo la opinión de la doctrina más autoritaria que, "el concepto de desarrollo sostenible se encuentra en algún lugar del espectro de políticas -no legales- y normas -legales-. Pudiendo quizás excluir los dos extremos". Por un lado, el concepto es más que un mero ideal político, como se ha mencionado repetidamente por la normativa jurídica internacional - por ejemplo, la Convención sobre el Cambio Climático de 1992, el artículo 3, el Convenio sobre la Diversidad Biológica de 1992, los artículos 8 y 10, 5, Convención de Antigua de 1992, artículo $2-{ }^{26}$ y por el derecho no vinculante -soft law-. Por otra parte, no podemos hablar de una verdadera norma jurídica, ya que carece de consecuencias legales directas. La proximidad a la política resulta más practicable, pero ¿en qué medida es más cercana? En este aspecto, es paradigmático para el derecho internacional el caso relativo al dique de Gabčikovo-Nagymaros - Hungría / Eslovaquia- ${ }^{27}$ en el que la CIJ utilizó como argumento del desarrollo sostenible en la resolución del mismo, pero con tal cautela y ambigüedad en la frase que no se puede hablar de un cambio firmemente establecido por la jurisprudencia en virtud del cual el desarrollo sostenible pueda convertirse en un elemento de interpretación hermenéutica para el tribunal en conflictos similares, aunque interpretaciones posteriores de la CIJ hayan seguido ese camino ${ }^{28}$. La pertinencia de este juicio es que, hay posibilidad de que el desarrollo sostenible pueda establecerse como norma jurídica vinculante en la comunidad internacional ${ }^{29}$, más como un objetivo político que como un principio legal ${ }^{30}$.

\section{Gobernanza global transformada por el paradigma de la sostenibilidad: Gobernanza ambiental global}

Rodrigo, tras analizar la práctica, la jurisprudencia internacional y la doctrina mayoritaria, concluye que este principio no es una norma de derecho internacional general, pero nos muestra como el principio de equidad intergeneracional que ha introducido aporta los elementos fundamentales a la noción de desarrollo sostenible, una dimensión temporal a largo plazo y una aspiración de justicia global que ha dado lugar a lo que algunos autores han Ilamado "política global del desarrollo"31. Por lo tanto, lo que es más importante señalar

\footnotetext{
${ }^{24}$ BOSSELMANN, Klaus, The principle of Sustainability... op.cit., p. 53. El autor postula por la sostenibilidad como principio, habla de su esencia con un contenido moral afirmado internacionalmente.

${ }^{25}$ En este punto veáse, LOWE, "Sustainable development... op.cit., p. 19.

${ }^{26}$ Para obtener más ejemplos véase, CORDONIER SEGGER, Marie Claire y KHALFAN, Ashfaq, Sustainable Development Law: Principles, Practices and Prospects, Oxford University Press, Oxford, 2004, p. 95.

${ }_{27}$ Asunto del dique Gabčikovo-Nagymaros, CIJ Informes 7, 1997, párrafo 140. Para un anális del caso en cuestión, LOWE, "Sustainable development... op.cit., p. 19 et seg.

${ }^{28}$ Arbitraje respecto a la Plancha Rin Ferrocarril (Bélgica v Holanda) Laudo del Rin de Hierro, PCA, 2005, párrafos 58-59; Asunto de las Plantas de celulosa sobre el río Uruguay (Pulp Mills case) (Medidas Provisionales) (Argentina v Uruguay), CIJ, informes, 2006, párrafo 80; Asunto de las plantas de celulosa sobre el río Uruguay (Méritos), CIJ Informes, 2010, párrafo 177.

29 Para un panorama detallado del concepto, BIRNIE, Patricia W. y BOYLE, Alan E., International Law and the Environment, Oxford University Press, Oxford, 1992, ps. 122-124 y LOWE, "Sustainable development... op.cit., supra note, p. 25 et seg.

${ }^{30}$ Ibídem, supra note.

${ }^{31}$ RODRIGO, Ángel J.., El desafío del desarrollo sostenible..... op.cit., p.36.
} 
aquí es en realidad la exitosa existencia del paradigma de sostenibilidad a nivel global. Las diferentes convenciones, como la Convención de las Naciones Unidas sobre el Cambio Climático (UNCCC), Kyoto y el Convenio sobre la Diversidad Biológica, demuestran la existencia de tal éxito.

Estos convenios internacionales muestran que, independientemente de los desafíos que estos acuerdos de cooperación representan para el escenario internacional y global, ya se está introduciendo lentamente un mejor marco de acción bajo los auspicios de la justicia social y la equidad. Tales acuerdos ofrecen una ilustración de la importancia de regímenes reguladores globalmente inclusivos guiados por principios jurídicos no reconocidos -legalmente- tales como el "principio de sostenibilidad". Otras evidencias importantes son los 17 ODS aprobados por la Asamblea General de la ONU en 2015, la COP 21 e COP 22, o incluso la jurisprudencia de la Corte Internacional de Justicia o el Órgano de Solución de Diferencias de la OMC, y las instituciones que la han invocado para la interpretación de tratados internacionales ${ }^{32}$.

Como resultado, es necesario asumir la existencia de un orden global que interacciona con una pluralidad de órdenes legales, y que posee una naturaleza fundamentalmente evolutiva. No obstante si comparamos el derecho ambiental internacional con otras áreas de derecho internacional -por ejemplo, la legislación de derechos humanos, el derecho internacional del trabajo o el derecho comercial internacional- podemos ver que está claramente poco desarrollado. No tiene un instrumento globalmente vinculante que establezca los derechos y obligaciones de los estados con respecto al medio ambiente. Sin embargo, la ausencia de un tratado internacional para la protección del medio ambiente a nivel mundial no ha determinado el fracaso de los procesos integradores de las preocupaciones ecológicas y la llegada de un escenario de derecho medioambiental global efectivo. Emerge en un contexto doctrinal que ha construido discursos teóricos acerca de la existencia de un derecho constitucional y administrativo global ${ }^{33}$. Según Elisa Morgera,

"[E]I concepto de ley ambiental global ayuda a comprender el papel 'funcional' de los estados y la 'funcionalización' de la soberanía nacional que surge de la evolución del derecho ambiental internacional en el contexto de la pluralidad de órdenes jurídicos. Los estados ejercen 'poderes delegados en interés de la humanidad en su conjunto'"'34.

Los estados, bajo estas premisas, están al servicio de la promoción de la justicia intergeneracional y el bienestar de los individuos y de ciertos grupos dentro de su propio territorio. Con este fin, el derecho ambiental mundial desempeña un papel crucial en la aplicación

32 Ibídem, p.39.

33 Véanse por ejemplo las obras de TWINING, William, Globalisation and Legal Scholarship, Cambridge University Press, Cambridge, 2000 y TWINING, William, General Jurisprudence: Understanding Law from a Global Perspective, Cambridge University Press, Cambridge, 2009; WALKER, Neil, "Beyond boundary disputes and basic grids: Mapping the global Disorder of Normative Orders" en International Journal of Constitutional Law, vol. 6, no 3-4, 2008, ps. 373-396; WALKER, Neil, "Constitutional pluralism in Global Context" en AVBELJ, Matej y KOMÁREK, Jan (eds.), Constitutional Pluralism in the European Union and Beyond, Hart, Oxford, 2012, ps. 17-38 y WALKER, Neil, Intimations of Global Law, Cambridge University Press, Cambridge, 2014.

34 MORGERA, Elisa, "Bilateralism at the Service of Community Interests? Non-judicial Enforcement of Global Public Goods in the Context of Global Environmental Law" en European Journal of International Law, vol. 23, no 3, 2012, ps. 743-767. 
del principio de responsabilidad común pero diferenciada en el derecho internacional ${ }^{35}$ que ha sido promovido con éxito por la CMNUCC y el Protocolo de Kyoto.

En este contexto la sostenibilidad surge como un "intento" de convertirse en un principio mundial, incluso la OMC ha dado pasos importantes hacia la protección del medio ambiente y la inclusión del principio de sostenibilidad en la dinámica de su programa de trabajo. El desarrollo sostenible es un objetivo de la OMC, como se reflejó en el Preámbulo del Acuerdo de Marrakech por el que se establece la OMC, y la jurisprudencia de la OMC ha contribuido a reforzar legalmente los principios de Nueva Delhi de desarrollo sostenible aunque ningún caso haya sido decidido usando como argumento el desarrollo sostenible propiamente dicho ${ }^{36}$.

Algunos tipos específicos de gobernanza mundial que conforman las bases de las futuras acciones legales nos ofrecen soluciones acerca de cómo la sostenibilidad y la protección "ecológica" podrían funcionar exitosamente. Esta "atractiva" construcción teórica de un emergente derecho global que surge ante la necesidad de superar los referentes institucionales tradicionales del derecho internacional -soberanía, territorialidad y estado nación, - tiene como fundamento "la internacionalización de los derechos humanos y la protección de la dignidad del individuo que permite a la sociedad civil desarrollarse frente a la hegemonía del estado", siguiendo esta línea de razonamiento es importante referirse a las obras de Krisch, Kingsbury y Stewart sobre The emergence of global administrative law ${ }^{37}$ y con particular importancia mencionar el derecho administrativo global en relación con cuestiones de derecho ambiental. El trabajo de los autores citados subraya la idea de la "protección ambiental" como una de las áreas que ha llevado a la creación y desarrollo del derecho administrativo global, subyacente en lo que es "el aumento en el alcance y las formas de regulación y administración transgubernamentales diseñadas para enfrentarse a las consecuencias de la interdependencia global"38. En lo que se refiere al denominado "espacio administrativo global"39 que sigue siendo rebatido ${ }^{40}$ por la doctrina jurídica en general, encontramos evidentes elementos de su afirmada existencia a través de varios ejemplos de regulación ambiental que surgen en la esfera de la regulación económica de la mando del Banco Mundial, OMC, OCDE, etc. y están proliferando bajo el paradigma de la sostenibilidad con el tratamiento diferenciado de la política climática ${ }^{41}$ donde subyace el principio de equidad intergeneracional.

${ }^{35} \mathrm{HEY}$, Ellen, "Global environmental Law and Global Institutions: A system lacking "'Good Process'" en PIERIK, Roland y WERNER, Wouter (eds), Cosmopolitanism in Context: Perspectives from International Law and Political Theory, Cambridge University Press, Cambridge, 2010, p. 50.

${ }^{36}$ En este punto véase, SCHURMANS, Marijke, "Sustainable Development is Emerging as a Core Tenet of WTO Case Law. To What Extent has it helped Enshrine this as a Legal Concept?" en European Energy and Environmental Law Review, vol. 24, n² 2, 2015, p. 28.

37 Véase KINGSBURY, Benedith; KRISCH, Nico y STEWART, Richard B., "The Emergence of Global Administrative Law" en Law \& Contemporary Problems, vol. 68, n 3-4, 2005, ps. 15-62.

${ }^{38}$ Ibíd.; en este punto también, CASINI, Lorenzo, "Diritto Amministrativo Globale" en CASSESE, Sabino (Dir.), Dizionario di Diritto Pubblico, Giuffrè, Milán, 2006.

39 Véase KINGSBURY, Benedith; KRISCH, Nico y STEWART, Richard B., "The Emergence of Global Administrative Law... op.cit.; CASSESE, Sabino, The Global Polity. Global Dimensions of Democracy and the Rule of Law, Global Law Press, Sevilla, 2012.

40 CASSESE, Sabino, "Global Administrative Law: The State of Art" en International Journal of Constitutional Law, vol. 13, no 2, 2015, ps.465-468.

${ }^{41}$ Muy debatido por la doctrina internacional, véase, Declaración de Río sobre el Medio Ambiente y el Desarrollo, A / CONF. 151/26 (volumen I), 1992, los principios 6-7. También RAJAMANI, Lavanya, Differentiated Treatment in International Environmental Law, Oxford University Press, Oxford, 2006. 
Además, un elemento significativo y distintivo del derecho global -lo que lo distingue del régimen clásico del derecho internacional- es que los sujetos del régimen regulatorio global son crecientemente los mismos que en el derecho interno: los individuos, empresas, grupos, asociaciones, e incluso $\mathrm{ONG}^{42}$. Un ejemplo magnífico son las normas ambientales - certificados - impuestas por el Banco Mundial para la concesión de ayuda a los países en desarrollo. Benedith Kingsbury et alt afirman que en ese

"espacio administrativo multifacético en el que los estados, los individuos, las empresas, las ONG y otros grupos o representantes de intereses sociales y económicos nacionales y mundiales se ven afectados por, la gobernanza reguladora global, interactúan de manera compleja 'permitiendo' la relativa autonomía y el carácter distintivo de este espacio administrativo global y de sus órganos decisorios cada vez más poderosos." ${ }^{43}$

En dicho espacio, bajo el paradigma de la sostenibilidad, han surgido innumerables iniciativas de cooperación en todos los niveles - que no discutiremos en profundidad-, algunos ejemplos son, el órgano de apelación de la OMC, el Programa 21, la COP 21, el mecanismo de "benefit-sharing" regulado por el Convenio sobre la diversidad biológica y la participación del público en los procesos de reglamentación. Las iniciativas locales son las que paradójicamente mejor muestran la importancia de aunar esfuerzos a nivel global. Un buen ejemplo de ello es el $11^{\circ}$ Objetivo de Desarrollo Sostenible sobre Desarrollo Urbano de la Agenda del desarrollo para el 2030; encontramos ciudades de todo el mundo comprometidas en hacer frente a los desafíos del desarrollo urbano integrando los principios de sostenibilidad en todas sus políticas locales y creando redes mundiales - C40 ciudades, Pacto de los Alcaldes que fue lanzado a nivel europeo en 2008 y extendido a países del Este, Mediterráneo, África subsahariana y en 2017 se espera el lanzamiento del Pacto Mundial de Alcaldes para el Clima y la Energía- ${ }^{44}$. Sin embargo, va más allá del alcance de este trabajo dedicarse a todas las iniciativas globales surgidas bajo los auspicios de la sostenibilidad, basta con decir que las fronteras entre los ámbitos políticos se están volviendo cada vez más borrosas. Existe una interconectividad horizontal cada vez mayor entre las esferas políticas, las finanzas, el comercio, la cultura, la seguridad, la paz y la cooperación que no pueden separarse del desarrollo sostenible -incluida la acción climática, la erradicación de la pobreza, la buena gobernanza, etc.- . En vez de instituciones separadas para tratar con ellos, hoy nos encontramos de frente a comunidades de diferentes actores y niveles de gobernanza que juntos logran crear un punto de encuentro global con un público heterogéneo y múltiple, e instituciones plurales ${ }^{45}$ que representan a la ciudadanía mundial.

42 Véase KINGSBURY, Benedith; KRISCH, Nico y STEWART, Richard B., "The Emergence of Global Administrative Law... op.cit., p.38. Los autores afirman: "[t]hat in such multifaceted administrative space where states, individuals, firms, NGOs, and other groups or representatives of domestic and global social and economic interests who are affected by, or otherwise have a stake in, global regulatory governance, interact in complex ways allowing the relative autonomy and distinct character of this global administrative space, and its increasingly powerful decision-making bodies".

${ }^{43}$ KINGSBURY, Benedith; KRISCH, Nico y STEWART, Richard B., "The Emergence of Global Administrative Law... op.cit.

${ }^{44}$ Consúltese http://www.eumayors.eu

45 STONE, Diane, "Global Public Policy, Transnational Policy Communities and their Networks" en Journal of Policy Sciences, vol. 36 no 10, 2008, ps. 19-38. 


\section{Una visión europea de la sostenibilidad: algunas cuestiones normativas}

Centrándonos en los avances ya descritos en relación a la naturaleza jurídica del concepto de sostenibilidad en el ámbito internacional, resulta fascinante observar el escenario Europeo. Sobre todo en términos de objetivos políticos efectivos hacía el desarrollo sostenible que toman la delantera en todas las áreas políticas de la UE y, por supuesto, en la relevancia de la sostenibilidad dentro de su marco constitucional que ha evolucionado hasta desligarse en gran parte de sus preocupaciones económicas originarías. La incorporación de este concepto en los tratados de la UE sigue reflejando una gran ambivalencia. Pero el principal desafío para la UE es superar el hecho de que su significado normativo no es el de un principio jurídico. Por lo tanto, el desarrollo sostenible no es legalmente justiciable porque no conduce a una obligación, que sería aplicable ante el CJUE. De hecho, las obligaciones vinculantes de naturaleza jurídica hacía el desarrollo sostenible son ampliamente más débiles que las de naturaleza política.

No obstante, esta contribución pretende mostrar cómo la noción europea de sostenibilidad, cuya herencia proviene del contexto global, ensambla perfectamente en el actual marco legal de la UE encajando dentro de las categorías tradicionales de normatividad. La primera premisa comienza por determinar cómo la UE sea un experimento único y exitoso en la gobernanza ambiental regional, especialmente después de haber visto la falta de una gobernanza operativa legalmente vinculante a nivel internacional y global. Esto es ampliamente aceptado desde el punto de vista de la gradual transferencia de los derechos soberanos de los estados al nivel supranacional de la UE ${ }^{46}$. Para algunos es notable cómo "en más de 50 años los estados miembros de la UE han notado las ventajas que se obtienen mediante la transferencia creciente de los derechos soberanos a un nivel supranacional". Por supuesto la ventaja evidente de esto es que los estados miembros coordinan sus esfuerzos mediante la legislación y la toma de decisiones centralizada. Por lo tanto, con este fin, la UE proporciona un modelo ejemplar de gobernanza para la sostenibilidad. A medida que la UE se ha ampliado, el número de estados europeos sujetos a la política de la UE y el impacto de la misma en materia de desarrollo sostenible ha aumentado a veintiocho estados miembros - dejando de lado los eventuales cambios graduales al contrario, "Brexit", etc.-, lo que significa que una mayor integración económica dentro de la UE, y entre la UE y otros estados europeos ha aumentado.

La UE ampliada ha ganado un mayor protagonismo y reputación en las convenciones y negociaciones internacionales relacionadas con la sostenibilidad y el desarrollo sostenible. Del mismo modo ha tenido una "aceptación inmediata del apelo a la sostenibilidad originada por el escenario internacional"47. La UE agregó nociones de sostenibilidad en su marco constitucional muy rápidamente. Los dos destacados episodios de esta adopción normativa de sostenibilidad en la UE como una forma incipiente de lo que son hoy en día han sido, el quinto Programa de Acción Ambiental (PAA) y el Tratado de Amsterdam sobre la UE. El primer PAA recoge la definición de desarrollo sostenible propuesta por la Comisión Brundtland con el objetivo de transformar los patrones de crecimiento dentro de la comunidad de la UE

\footnotetext{
${ }^{46}$ BOSSELMAN, Klaus, The principle of Sustainability... op.cit., p.187.

${ }^{47}$ Ibíd., p. 188.
} 
para poder promover la sostenibilidad ${ }^{48}$. El Tratado de Amsterdam, por el que se modifica el Tratado de la Unión Europea, fue el primer texto legal que introdujo la noción y abarca la definición aceptada internacionalmente de desarrollo sostenible, en la que se proclama que "la Comunidad tendrá por misión promover un desarrollo armonioso, equilibrado y sostenible de las actividades económicas"49 vinculadas al desarrollo sostenible. Por lo tanto, dentro del marco constitucional de la UE, el desarrollo sostenible fue inicialmente introducido a través del "principio de integración"50. Dicho principio está ahora consagrado en el artículo 11 del Tratado de Funcionamiento de la Unión Europea (TFUE), que prevé la protección del medio ambiente mediante la inclusión de consideraciones ambientales en la primera fase del proceso de desarrollo.

Pero la sostenibilidad fue introducida prominentemente por el Tratado de Lisboa. El concepto está consagrado actualmente en las diversas disposiciones del Tratado, los apartados 3 y 5 del artículo 3 del TUE, el artículo 21, apartado 2, letras d) y f), el artículo 11 del TFUE, así como el artículo 37 de la Carta Europea de Derechos Fundamentales. Las definiciones que están consagradas en los distintos instrumentos que representan el derecho primario de la UE y que enmarcan el escenario constitucional de la UE no se refieren exclusivamente al desarrollo de las actividades económicas. El artículo 3, apartado 3, del TUE dice lo siguiente: "La Unión ... obrará en pro del desarrollo sostenible de Europa basado en un crecimiento económico equilibrado y en la estabilidad de precios, en una economía social de mercado altamente competitiva, dirigida al pleno empleo y al progreso social. Alto nivel de protección y mejora de la calidad del medio ambiente. Promoverá el avance científico y tecnológico".

El objetivo clave ha sido vincular el desarrollo económico, la protección del medio ambiente y la justicia social. Sin embargo, es importante recordar que el desarrollo sostenible, así como la protección del medio ambiente, no pueden separarse del mercado interno, independientemente de la evolución del concepto hacia un carácter conciliador ${ }^{51}$ que también se refleja en un acervo de instrumentos de derecho derivado perteneciente a un acquis communitaire aún no reconocido abiertamente ${ }^{52}$. La introducción de tal concepto, en sus diversas formas, por el marco constitucional de la UE, significa un paso adelante cuando se

${ }^{48}$ Véase el 50 Programa Ambiental Europeo. Disponible en http://europa.eu/scadplus/leg/en/lvb/128062.htm [consultado el 20 de diciembre de 2016]

${ }^{49}$ Véase, para la síntesis e historia del concepto de "desarrollo sostenible" en el derecho de la UE , KRÄMER, Ludwig, "Sustainable development in EC law" en BUGGE, Hans Christian y VOIGT, Christina (eds.), Sustainable development in international and national law, Europa Law Publishing, Groningen, 2008, ps. 377-379.

50 El principio de integración posee encuentra sus raíces en el importante caso de las botellas Danesas, véase, Asunto 302/86, EC Commission v Denmark, 1 CMLR 619, 1989.

${ }^{51}$ La primera aparición de tal naturaleza conciliatoria vino de la mano de un dictámen de la CEJ, First Corporate Shipping, un caso sobre desarrollo en un contexto de hábitat de aves protegidas, fue testimonio de una interpretación conciliadora. Véase, Asunto C-371/98, First Corporate Shippping, E.C.R. I-9235, 2000, párrafo 54 (opinión AG Léger).

52 Es posible identificar estos aspectos de la política de sostenibilidad en la UE a través de las iniciativas de los Programas Ambientales Europeos, el primero de los cuales fue adoptado en 1972, y seguido ininterrumpidamente por otros, 1977-1982, 1982-1987, 1987-1992, 1992-2002, and 2002-2012, 2013-2020. No obstante, hasta el 50 Programa (1992-2000) no se introdujo una cercanía hacía una fuerte posición promotora de "sostenibilidad" y un comprometido programa de acción hacía el desarrollo sostenible. Véanse capítulos 2 y 13 de BARNES, Pamela, M. y HOERBER, Thomas, C. (eds) Sustainable development and Governance... op.cit. El séptimo programa ambiental, "Living well within the limits of our planet", muestra un compromiso aún mayor hacía una visión holística de sostenibilidad proclamando un acercamiento ecocéntrico, que comporta justicia social y "good governance". 
tienen en cuenta los imperativos de preservación de la naturaleza, independientemente de un enfoque fragmentado en la legislación secundaria - estableciendo diferentes criterios rectores para la sostenibilidad en agricultura, energía, recursos energéticos renovables, etc-. Además, de acuerdo con el apartado 5 del artículo 3 del TUE, así como del artículo 21, apartado 2, letra d), del TUE, el desarrollo sostenible se convierte también en una de las piedras angulares de La política exterior de la UE. La obligación de tener en cuenta el desarrollo sostenible en las relaciones internacionales de la UE adquiere una importancia notable ${ }^{53}$.

Por otra parte, el artículo 11 del TFUE vuelve a proponer el principio de integración, establece que: "Los requisitos de protección del medio ambiente deben integrarse en la definición y aplicación de las políticas y actividades de la Unión, en particular con vistas a promover el desarrollo sostenible diferente de esta formulación, el artículo 37 de la Carta de Derechos Fundamentales de la UE, dice así, "Un alto nivel de protección ambiental y de mejora de la calidad del medio ambiente debe integrarse en las políticas de la Unión y garantizarse de acuerdo con el principio de desarrollo sostenible". Una vez dicho esto, es importante afirmar que el hecho de que el desarrollo sostenible se condense en tres disposiciones diferentes situadas en la parte superior de la pirámide jerárquica del ordenamiento jurídico de la UE no significa que su estatuto jurídico no se desvanezca por las controversias. Tal y como lo expresa Nicolas de Sadeleer, el desarrollo sostenible tiene una "situación atormentada por las controversias" 54 , lo que provoca quizás algunos problemas en lo que respecta a la interpretación conceptual y su estatus, conduciendo a una cierta reticencia de las instituciones de la Unión Europea -especialmente la CJUE- para desarrollar y valerse del concepto como instrumento hermenéutico para la resolución de los conflictos y la interpretación jurisprudencial. Hay un vacío en lo que se refiere a casos cuyo dictamen haya sido determinado por el paradigma de la sostenibilidad, no obstante, en jurisprudencia reciente, la corte ha abordado la cuestión del desarrollo sostenible en relación con la naturaleza vinculante del mismo en lo que se refiere a la cooperación internacional de la UE después de Lisboa ${ }^{55}$, y para definir su relevancia en la interpretación de sus políticas sectoriales ${ }^{56}$.

¿Podría la sostenibilidad convertirse realmente en un concepto normativo dentro de la legislación de la UE? Autores como Sadeleer no tienen problemas en afirmar que "unido a las exigencias de la integración, un alto nivel de protección y los diferentes principios del derecho ambiental - prevención, precaución, contaminación, etc. - el desarrollo sostenible se ha convertido en un concepto normativo" ${ }^{\prime 57}$ Es indudablemente un objetivo constitucional vinculante. Por lo tanto, en lugar de ser escépticos acerca de la posibilidad de que la sostenibilidad represente un paradigma primario de las numerosas políticas de la UE, debemos mirar su

\footnotetext{
53 En este punto véase, MARIN-DURAN, Gracia y MORGERA, Elisa, Environmental Integration in the EU's External Relations, Modern Studies in European Law, vol. 29, Hart, UK, 2012. Ver el caso más reciente del Tribunal Europeo de Justicia en este aspecto.

${ }^{54}$ SADELEER, Nicolas de, "Sustainable Development in EU Law: still a long way to go" en Jindal Global Law Review, vol. 6, no 1, 2015, ps. 39-60.

${ }^{55}$ C-377/12 Comisión Europea / Consejo de la Unión Europea (Filipinas PCFA) UE: C: 1903, Sentencia del Tribunal Europeo de Justicia, 2014.

${ }^{56}$ C-461/13, Bund v Alemania, ECLI: UE: C: 2324 (opinión AG Jääskinen), 2014. También véase, PALONIITTY, Tiina, "The Weser Case: Case 461/13 Bun v Germany" en Journal of Environmental Law, vol. 28, no 1, 2016, ps. 151-158.

${ }^{57}$ SADELEER, Nicolas de, "Sustainable Development in EU Law... op.cit., p. 59.
} 
rápida evolución y el papel crucial de la UE tratando de coordinar sus 28 estados miembros para luchar por problemas ecológicos, el suministro de energía, el desarrollo tecnológico y las iniciativas locales de buen gobierno como el "Pacto de los Alcaldes". Hay otro aspecto que no debemos olvidar que surge alrededor de 2007, relacionado con la "'green' economy" de bajo carbono, centrada en la creación de empleo. Se convirtió en un punto crucial de los debates políticos ${ }^{58}$ y recibió el apoyo del Parlamento Europeo, la Comisión Europea y algunos de los Estados miembros de la UE, en particular Alemania y el Reino Unido. Para lograr la economía baja en carbono o "green economy", son necesarias tres cosas: la protección del medio ambiente, la creación de empleo y el abastecimiento seguro de energía como "[T]he entire industrial infrastructure, based on the back of fossil fuels, is aging and in disrepair" ${ }^{\prime 59}$. Rifkin argumentó que poner el medio ambiente en el centro de la economía ayudaría a lograr el uso sostenible de los recursos naturales. Como resultado, habría una protección del medio ambiente, una seguridad energética y unos beneficios económicos al pasar a una "green economy". Sin embargo, mientras el discurso sobre la economía verde ha ganado en apoyo - es decir, el gobierno italiano ha promulgado una ley siguiendo esta tendencia conceptualno ha reemplazado al de desarrollo sostenible como discurso hegemónico; más bien, parece apoyar el discurso que identifica una agenda de acción en aras del desarrollo sostenible.

El ejemplo de la política energética de la UE pone de manifiesto la manera en que el paradigma integral del desarrollo sostenible ha sido de alguna manera sustituido por una definición más "reducida" de sostenibilidad. Demasiado a menudo se ha percIbído la sostenibilidad como la dimensión de la política energética centrada simplemente en la reducción de gases invernadero para combatir el cambio climático. En cambio, es a través del proceso de formulación de políticas energéticas a nivel de la UE ${ }^{60}$, y los cambios significativos introducidos a nivel constitucional en este campo que el paradigma de la sostenibilidad llega al centro de atención de la UE fomentando un nuevo acquis communitaire que propugna un "Sustainable European making".

No obstante, aunque la política energética ha sido uno de los campos más prominentes que abarcan el paradigma de la sostenibilidad en Europa, es también, de una manera controvertida, el que posee más riesgos de construir su marginación centrándose en el interés de los estados miembros en la descarbonización sin llegar a políticas comunes o la transmisión de buenas prácticas que estabilicen la posición hegemónica de las demandas sociales y el desarrollo sostenible ${ }^{61}$. En cualquier caso, es posible decir, considerando iniciativas concretas a nivel europeo - sobre energía, comercio, desarrollo agrícola, ordenación del territorio, etc. - o

${ }^{58}$ Así RIFKIN, Jeremy, The Third Industrial Revolution: how Lateral Power is Transforming Energy, the Economy and the World, Palgrave Macmillan, Nueva York, 2011.

${ }^{59}$ Ibíd., p. 1.

${ }^{60}$ Cabe destacar aquí, el proceso de Cardiff, el llamado proceso de Cardiff - sin importar su fracaso-, lanzado en 1998, se refiere a la integración del medio ambiente en las políticas sectoriales como la energía en aras del desarrollo sostenible. A pesar de varias cumbres sobre el proceso de Cardiff con miras a desarrollar una estrategia integral e integrada para la sostenibilidad, sigue sin estar claro cómo y en qué medida el proceso de Cardiff puede vincularse a la agenda de la sostenibilidad. Además, el Tratado de Lisboa introduce un capítulo sobre la energía. La política energética surge "completamente nueva" en el dominio soberano de la UE, lo que sugiere en su redacción "sostenibilidad" con carácter conciliador respecto de algunas preocupaciones ecológicas, recogidas en los artículos 194 y 195 del TFUE.

${ }^{61}$ En este punto véase SÁNCHEZ GALERA, María Dolores, "La integración de las políticas energéticas y ambientales en la Unión Europea: Paradojas y Contradicciones a la luz del paradigma de la sostenibilidad" en Revista General de Derecho Administrativo, no 43, 2016. 
nacionales -es decir, la Ley francesa de transición energética de julio de 2015, La eliminación de la energía nuclear por parte de Alemania- que la UE va por el buen camino para mejorar el bienestar social y medioambiental dentro de sus estados miembros, independientemente de las raíces económicas de sus tratados originarios propugnando un buen funcionamiento de mercado. Hoy en día, la UE puede exportar un modelo de gobernanza basado en el paradigma de la sostenibilidad que es único. Los instrumentos jurídicos más importantes de este modelo que conformarán el futuro acquis communitaire en relación con la política sostenible están representados por el exitoso sistema europeo de Clima y Energía. La UE es el líder mundial en la política climática, ya que ya ha dado pasos importantes hacia un futuro sostenible y de eliminación del carbono. El reciente Energy Road Map 2050, tiende a favorecer la eliminación completa -80 a 95 por ciento de reducción- de los gases de efecto invernadero a mediados de siglo. Los objetivos específicos hasta el 2020 son los siguientes: una reducción del 20\% de los gases de invernadero de la UE a partir de los niveles alcanzados en 1990; alcanzar un $20 \%$ de producción energética a través del uso de fuentes de energía renovables; y un $20 \%$ de mejoría de la eficiencia energética. Las fuentes normativas principales son la Directiva sobre Energías Renovables 2009/28/EC - una nueva nos espera en el umbral de la puerta-, la Directiva de Eficiencia Energética, y el paquete "aire puro" para mejorar la calidad del aire en Europa. A nivel de la UE en su conjunto, el Sistema de Comercio de Derechos de Emisión de la UE -ETS de la UE- está trabajando en el principio de "cap and trade" y actualmente, está en su tercera fase (2013-2020) implementando un único límite de contaminación para emisiones que es aplicable en sustitución de los antiguos sistemas de límites nacionales.

Los instrumentos normativos y la cantidad ingente de legislación vinculante, comunicaciones, recomendaciones, etc., procedentes de la Comisión de la UE han conseguido que el conjunto de leyes relacionadas con la protección del medioambiente, el cambio climático y el suministro de energía se conviertan en un área normativa extremadamente compleja y tremendamente ambiciosa. A través de este ambicioso conjunto de instrumentos normativos un éxitoso paradigma de desarrollo sostenible está construyendo un nuevo cuerpo de acquis communitaire en materia de protección del medio ambiente capaz de integrar todas las políticas sectoriales europeas.

\section{Conclusiones}

La sostenibilidad sin duda es un nuevo paradigma de acción, ya sea a nivel internacional, regional o local. El esfuerzo de "integración" y "equilibrio" que exige el desarrollo sostenible no puede sacrificar bajo ningún pretexto la integridad ecológica para tener éxito, especialmente en un mundo - todavía - fuertemente conceptualizado en términos económicos. Su naturaleza proactiva y su existencia normativa dinámica y contextual han llevado a la doctrina reciente a enfatizar el alcance de su dimensión social como un requisito "abierto" de desarrollo que acoge una amplia gama de dimensiones hasta llegar a incluir, paz, seguridad, equidad, 62 e incluso cultura y ha determinado su existencia prominente eclipsando la noción de "green economy".

Independientemente de su éxito al alcanzar una posición hegemónica en un contexto global crecientemente proclive a la conciliación de todas las políticas sectoriales y al

62 RODRIGO, Ángel J., El desafío del desarrollo sostenible....op.cit.. p. 39. 
acercamiento de todos los niveles de gobierno y de todos los procesos ligados a las prácticas de buen gobierno, no parece haber un claro reconocimiento de su carácter vinculante, ni de su condición como principio jurídico reconocido por el derecho internacional. Con lo cual, en un contexto mundial liderado por una pluralidad de actores, el desarrollo sostenible resulta ser el paradigma a través del cual las complejas redes de relaciones alcanzan un diálogo de acción integrando funciones separadas. Las relaciones de dicho contexto se nutren de la diversidad de actores que incluyen las instituciones globales, organizaciones regionales, estados, entidades regionales subnacionales, organizaciones no gubernamentales, redes políticas transnacionales y sociedad civil.

Esta nueva conceptualización de la gobernanza, la ciudadanía y el diálogo en las relaciones internacionales auspiciada por la sostenibilidad presupone una multiplicidad de ciudadanos con un estatus político-legal - posnacionalismo-, y un reconocimiento de identidades diversas y múltiples - multiculturalismo versus interculturalismo-y la participación ciudadana en todos los niveles de soberanía -es decir, transnacionalismo-. Además, la creciente concienciación sobre la necesidad del conocimiento global y la planificación global para la protección de bienes globales, así como el reconocimiento de un futuro compartido, beneficia el interés por los valores universales de pertenencia y la expresión institucional de las normas globales que favorecen la consecución de los objetivos de desarrollo sostenible.

Por otra parte, el debate sobre su sustancia conceptual y su naturaleza jurídica sigue abierto, pero existe un consenso en cuanto a su ambición por integrar las cuestiones sociales, económicas, medioambientales y de buen gobierno. La naturaleza multidimensional del concepto y su materialización como objetivo político quedan satisfechas hoy por una amplia gama de valores que han situado el desarrollo sostenible entre los objetivos imperativos de la ONU, la paz y la seguridad, con claras deficiencias en los mecanismos impositivos.

La Estrategia de Desarrollo Sostenible de la UE proporciona un marco adecuado para analizar la naturaleza evolutiva de la noción en el mundo "desarrollado" y la eficacia de un concepto que se nutre de una esencia global pero alcanza su máxima eficacia operando a nivel regional. El significado y la fuerza jurídica de la sostenibilidad a escala europea, constitucionalmente aplicados - aunque tiene un largo camino por recorrer para fomentar un desarrollo auténticamente sostenible $-{ }^{63}$ se han convertido en un modelo único de gobernanza eficaz para implementar el desarrollo sostenible a través de políticas e instrumentos vinculantes que podrían cambiar la situación futura a través de una política de cambio climático y eficiencia energética exitosa que va más allá de los intereses de integración de mercado. No obstante, a pesar de la "retórica" de la Comisión Europea sobre sostenibilidad y la centralidad de las preocupaciones sobre el cambio climático no es tan fácil lograr una integración efectiva de los objetivos ambientales combinados con los criterios de sostenibilidad de otras políticas sectoriales, como la industrial, la energética, etc. Sin embargo, el nuevo paradigma de desarrollo sostenible que está favoreciendo la construcción de un complejo acquis communitaire en materia de protección del ambiente rige todas las políticas sectoriales europeas y está aquí para quedarse. La CJUE, que carece de una hermenéutica interpretativa

${ }^{63}$ Véase el análisis de Barnes, Hoerber y otros, sobre la evolución del concepto en Europea y su implementación en las políticas sectoriales más influyentes, BARNES \& HOERBER (eds) Sustainable development and Governance in Europe, 2013, Routledge. 
en base a la sostenibilidad, podría desempeñar en el futuro un papel importante en los asuntos relacionados con el cumplimiento de las normas obligatorias de sostenibilidad, o los mandatos relacionados que todavía faltan en la actualidad. El futuro está ya en nuestras manos.

\section{Bibliografía}

ARIAS MALDONADO, Manuel, Environment and Society: Socionatural relations in the Anthropocene, Springer, 2015.

BAKKER, Christine y FRANCIONI, Francesco, The EU, the US and Global Climate Governance, Routledge, Londres y Nueva York, 2014.

BARNES, Pamela, M. y HOERBER, Thomas, C. (eds) Sustainable development and Governance in Europe, Routledge, Londres y Nueva York, 2013.

BARNES, Pamela M. y HOERBER, Thomas, C., "Linking the discourse on Sustainability and Governance" en BARNES, Pamela, M. y HOERBER, Thomas, C. (eds) Sustainable development and Governance in Europe, Routledge, Londres y Nueva York, 2013.

BARRAL, Virginie, "Sustainable Development in International Law: Nature and Operation of an Evolutive Legal Norm" en European Journal of International Law, vol. 23, no 2, ps. 377-400.

BECK, Ulrich, What is globalisation?, Polity Press, Cambridge, 2007.

BENVENISTI, Eyal, The Law of Global Governance, Hague Academy of International Law, La Haya, 2014 BIRNIE, Patricia W. y BOYLE, Alan E., International Law and the Environment, Oxford University Press, Oxford, 1992.

BOSSELMAN, Klaus, The Principle of Sustainabaility. Transforming Law and Governance, Ashgate, Farnham, 2008.

BOSSELMANN, Klaus, "Sustainability and the Courts: A Journey Yet to Begin?" en Journal of Court Innovation, vol. 3, no 1, 2010, p. 338.

BOYLE, Alan y FREESTONE, David (eds) International Law and Sustainable Development. Past Achievements and Future Challenges, Oxford University Press, Oxford, 1999.

CARADONNA, Jeremy L., Sustainability. A History, Oxford University Press, Oxford, 2014.

CARLOWITZ, Hans C. von, Sylvicultura oeconomica, oder haußwirthliche Nachricht und Naturmäßige Anweisung zur wilden Baum-Zucht, 1713 [edición de Leipzig, repr. Freiberg, TU Bergakademie Freiberg und Akademische Buchhandlung, 2000].

CASINI, Lorenzo, "Diritto Amministrativo Globale" en CASSESE, Sabino (Dir.), Dizionario di Diritto Pubblico, Giuffrè, Milán, 2006.

CASSESE, Sabino, The Global Polity. Global Dimensions of Democracy and the Rule of Law, Global Law Press, Sevilla, 2012.

CASSESE, Sabino, "Global Administrative Law: The State of Art" en International Journal of Constitutional Law, vol. 13, no 2, 2015, ps.465-468.

COMISIÓN MUNDIAL SOBRE MEDIO AMBIENTE Y DESARROLLO (UNWCED), Nuestro Futuro Común. Brundtland Report, Oxford University Press, Oxford y Nueva York, 1987.

CORDONIER SEGGER, Marie Claire y KHALFAN, Ashfaq, Sustainable Development Law: Principles, Practices and Prospects, Oxford University Press, Oxford, 2004.

ESTY, Daniel C. e IVANOVA, Maria, "Globalisation and Environmental Protection: A Global Governance Perspective", Working Paper 402, 21 de Julio de 2004. Disponible en https://pdfs. semanticscholar. org/6768/00768d0d2c7063bdd70466aa5ed731066f81.pdf [consultado el 26 de enero de 2017]

EVELYN, John, Sylva, or a Discourse of Forest-Trees and the Propagation of Timber in His Majesties Dominions, John Martyn, Londres, 1664.

MARIN-DURAN, Gracia y MORGERA, Elisa, Environmental Integration in the EU's External Relations, Modern Studies in European Law, vol. 29, Hart, UK, 2012.

GROBER, Ulrich Deep Roots. A Conceptual History of "Sustainable Development" (Nachhaltigkeit), 
Wissenschaftszentrum Berlin für Sozialforschung (WZB), Berlin, Febrero, 2007.

HARDIN Garrett, "The Tragedy of the Commons" en Science, vol. 162, no 3859, Diciembre, 1968 , ps. 1243-1248.

HEY, Ellen, "Global environmental Law and Global Institutions: A system lacking "'Good Process'" en PIERIK, Roland y WERNER, Wouter (eds), Cosmopolitanism in Context: Perspectives from International Law and Political Theory, Cambridge University Press, Cambridge, 2010, ps. 4573.

KRÄMER, Ludwig, "Sustainable development in EC law" en BUGGE, Hans Christian y VOIGT, Christina (eds.), Sustainable development in international and national law, Europa Law Publishing, Groningen, 2008, ps. 377-398.

KINGSBURY, Benedith; KRISCH, Nico y STEWART, Richard B., "The Emergence of Global Administrative Law" en Law \& Contemporary Problems, vol. 68, no 3-4, 2005, ps. 15-62.

LOPERANA, Demetrio, Desarrollo Sostenible y Globalización, Aranzadi, 2003.

LOWE, Vaughan, "Sustainable development and Unsustainable arguments" en BOYLE, Alan y FREESTONE, David (eds) International Law and Sustainable Development: Past Achievements and Future Challenges, Oxford University Press, Oxford, 1999.

McAFFE, Kathleen, "Selling nature to save it? Biodiversity and the rise of green developmentalism" en Environment and Planning, vol. 17, no 2, 1999, ps. 133-154.

MORGERA, Elisa, "Bilateralism at the Service of Community Interests? Non-judicial Enforcement of Global Public Goods in the Context of Global Environmental Law" en European Journal of International Law, vol. 23, no 3, 2012, ps. 743-767.

NACIONES UNIDAS, Prototype Global Sustainable Development Report, Departamento de Asuntos Económicos y Sociales, División de Desarrollo Sostenible, Nueva York, Julio 2014. Disponible en https://sustainabledevelopment.un.org/globalsdreport/ [consultado el 26 de enero de 2017]

PALONIITTY, Tiina, "The Weser Case: Case 461/13 Bun v Germany" en Journal of Environmental Law, vol. 28, no 1, 2016, ps. 151-158.

PROGRAMA DE NACIONES UNIDAS PARA EL MEDIO AMBIENTE (UNEP), Towards a Green Economy; pathways to sustainable development and poverty eradication - a Sinthesis for Policy Makers, 2011. Disponible en https://sustainabledevelopment.un.org/content/documents/126GER_ synthesis_en.pdf [consultado el 1 de Febrero de 2017]

RADKAU, Joachim, Natur und Macht. Eine Weltgeschichte der Umwelt, Beck, Munich, 2000

RAJAMANI, Lavanya, Differentiated Treatment in International Environmental Law, Oxford University Press, Oxford, 2006.

RIFKIN, Jeremy, The Third Industrial Revolution: how Lateral Power is Transforming Energy, the Economy and the World, Palgrave Macmillan, Nueva York, 2011.

ROBINSON, John, "Squaring the Circle? Some thoughts on the Idea of Sustainable Development" en Ecological Economics, vol. 48, no 4, 2004, ps. 369-384.

RODRIGO, Ángel J., "El principio de integración de los aspectos económicos, sociales y medioambientales del desarrollo sostenible" en Revista Española de Derecho Internacional, vol. LXIV, no 2, 2012, ps. $133-161$.

RODRIGO, Ángel J., El desafío del desarrollo sostenible. Los principios de Derecho internacional relativos al desarrollo sostenible, Centro de Estudios Internacionales, Marcial Pons, Madrid, 2015.

SACHS, Jeffrey D., The Age of Sustainable Development, Columbia University Press, Nueva York, 2015. SADELEER, Nicolas de, Environmental Principles. From Political Slogans to Legal Rules, Oxford University Press, Oxford, 2002.

SADELEER, Nicolas de, "Sustainable Development in EU Law: still a long way to go" en Jindal Global Law Review, vol. 6, no 1, 2015, ps. 39-60.

SÁNCHEZ GALERA, María Dolores, "La integración de las políticas energéticas y ambientales en la Unión Europea: Paradojas y Contradicciones a la luz del paradigma de la sostenibilidad" en Revista General de Derecho Administrativo, no 43, 2016.

SANDS, Peel, "Environmental Protection in the Twentieth Century: Sustainable Development and 
International Law" en VIG, Norman J. y AXELROLD, Regina S. (eds.), The Global Environment. Institutions, Law and Policy, Earthscan, Washington D.C., 1999, ps. 116-137.

SANDS, Peel, "Global Environmental Change and the Nation State" en Winter, G. (ed.), Multilevel Governance of Global Environmental Change, Cambridge University Press, Cambridge, 2006, ps. 519-538.

SANDS, Peel, Principles of International Environmental Law, Cambridge University Press, Cambridge, 2003.

SANZ LARRUGA, Francisco J., "Environmental Law and its Relationship with Global Administrative Law" en ROBALINO-ORELLANA, Javier y RODRíGUEZ-ARANA, Jaime (eds.), Global Administrative Law. Towards a Lex Administrativa, Cameron May, Londres, 2010.

SARASÍBAR, Miren, Régimen Jurídico del Cambio Climático, Lex Nova, Valladolid, 2006.

SCHURMANS, Marijke, "Sustainable Development is Emerging as a Core Tenet of WTO Case Law. To What Extent has it helped Enshrine this as a Legal Concept?" en European Energy and Environmental Law Review, vol. 24, no 2, 2015, ps. 28-34.

STEWART, R.B., "Addressing problems of disregard in global regulatory governance: Accountability, participation and responsiveness", Working Paper 2014/2 (Global Administrative Law Series), Institute for International Law and Order, 7 de noviembre de 2014. Disponible en http://www. iilj.org/wp-content/uploads/2016/08/Stewart-Addressing-Problems-of-Disregard-in-GlobalRegulatory-Governance-IILJ-WP-2014_2-GAL.pdf [consultado el 27 de enero de 2017]

STIGLITZ, Joseph E., Globalization and its Discontents, Norton Paper Back, Nueva York, 2003.

STONE, Diane, "Global Public Policy, Transnational Policy Communities and their Networks" en Journal of Policy Sciences, vol. 36 no 10, 2008, ps. 19-38.

TAYLOR, Prue y BOSSELMANN, Klaus, "The Earth Charter in the Classroom: Transforming the Role of Law" en Education for Sustainable Development in Action-Good Practices Using the Earth Charter, UNESCO y Earth Charter International, San José de Costa Rica, 2007.

TWINING, William, Globalisation and Legal Scholarship, Cambridge University Press, Cambridge, 2000. TWINING, William, General Jurisprudence: Understanding Law from a Global Perspective, Cambridge University Press, Cambridge, 2009.

WALKER, Neil, "Beyond boundary disputes and basic grids: Mapping the global Disorder of Normative Orders" en International Journal of Constitutional Law, vol. 6, no 3-4, 2008, ps. 373-396.

WALKER, Neil, "Constitutional pluralism in Global Context" en AVBELJ, Matej y KOMÁREK, Jan (eds.), Constitutional Pluralism in the European Union and Beyond, Hart, Oxford, 2012, ps. 17-38.

WALKER, Neil, Intimations of Global Law, Cambridge University Press, Cambridge, 2014.

YOUNG, Oran (ed), Global Governance: Drawing Insights from the Environmental Experience, MIT Press, Cambridge, Massachusetts, 1997.

\section{Capítulos, Resoluciones, Informes, Tratados}

Carta de Aalborg de las Ciudades Europeas hacía la Sostenibilidad, 1994.

Declaración de Johannesburgo sobre el Desarrollo Sostenible (Declaración de Johannesburgo) Johannesburgo, 4 de septiembre de 2002, UN Doc. A / Conf. 199/20.

Declaración de Nueva Delhi sobre los principios del derecho internacional relacionados con el desarrollo sostenible, resolución ILA 3/2002, Londres, 2002.

Declaración de Río sobre el Medio Ambiente y el Desarrollo, A / CONF. 151/26 (volumen I), 1992.

Informe de la Cumbre Mundial Sobre Desarrollo Sostenible, Johannesburgo, 26 de Agosto al 4 de Septiembre de 2002. Disponible en http://www.cepal.org/rio20/noticias/paginas/6/43766/ WSSD_Informe.ESP.pdf [consultado el 27 de Enero de 2017]

Programas de Acción Ambiental de la Ue (PAA). El primero fue adoptado en 1972 (1972-1977), y fue seguido por otros en 1977-1982, 1982-1987, 1987-1992, 1992-2002, 2002-2012 y 20132020.

Resolución S-19/2 de la Asamblea General de la ONU, 28 de junio de 1997.

Tratados constitutivos de las Comunidades Europeas y actos conexos, DO C340, 1997. 
50 Programa Ambiental Europeo. Disponible en http://europa.eu/scadplus/leg/en/lvb/128062.htm [consultado el 20 de diciembre de 2016]

\section{Jurisprudencia}

Arbitraje respecto a la Plancha Rin Ferrocarril (Bélgica v Holanda) Laudo del Rin de Hierro, PCA, 2005, párrafos 58-59.

Asunto C-371/98, First Corporate Shippping, E.C.R. I-9235, 2000, párrafo 54 (opinión AG Léger).

Asunto del dique Gabčikovo-Nagymaros, CIJ Informes 7, 1997, párrafo 140.

Asunto de las Plantas de celulosa sobre el río Uruguay (Pulp Mills case) (Medidas Provisionales) (Argentina v Uruguay), CIJ, informes, 2006, párrafo 80.

Asunto de las plantas de celulosa sobre el río Uruguay (Méritos), CIJ Informes, 2010, párrafo 177.

Asunto 302/86, EC Commission v Denmark, 1 CMLR 619, 1989.

C-377/12 Comisión Europea / Consejo de la Unión Europea (Filipinas PCFA) UE: C: 1903, Sentencia del Tribunal de Justicia, 2014.

C-461/13, Bund v Alemania, ECLI: UE: C: 2324 (opinión AG Jääskinen), 2014. 


\section{RELACIONES INTERNACIONALES}

Revista académica cuatrimestral de publicación electrónica Grupo de Estudios de Relaciones Internacionales (GERI) Universidad Autónoma de Madrid, España

www.relacionesinternacionales.info

ISSN 1699 - 3950

ff facebook.com/RelacionesInternacionales

twitter.com/RRInternacional 